\title{
Why choose Random Forest to predict rare species distribution with few samples in large undersampled areas? Three Asian crane species models provide supporting evidence
}

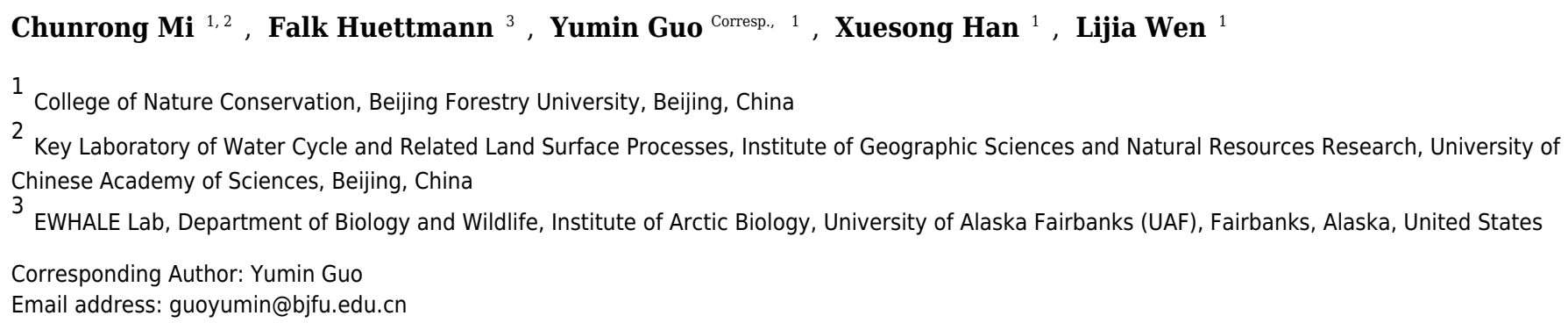

Species distribution models (SDMs) have become an essential tool in ecology, biogeography, evolution, and more recently, in conservation biology. How to generalize species distributions in large undersampled areas, especially with few samples, is a fundamental issue of SDMs. In order to explore this issue, we used the best available presence records for the Hooded Crane (Grus monacha, $n=33$ ), White-naped Crane (Grus vipio, $n=40$ ), and Black-necked Crane (Grus nigricollis, $n=75$ ) in China as three case studies, employing four powerful and commonly used machine learning algorithms to map the breeding distributions of the three species: TreeNet (Stochastic Gradient Boosting, Boosted Regression Tree Model), Random Forest, CART (Classification and Regression Tree) and Maxent (Maximum Entropy Models) Besides, we developed an ensemble forecast by averaging predicted probability of above four models results. Commonly-used model performance metrics (Area under ROC (AUC) and true skill statistic (TSS)) were employed to evaluate model accuracy. Latest satellite tracking data and compiled literature data were used as two independent testing datasets to confront model predictions. We found Random Forest demonstrated the best performance for the most assessment method, provided a better model fit to the testing data, and achieved better species range maps for each crane species in undersampled areas. Random Forest has been generally available for more than 20 years, and by now, has been known to perform extremely well in ecological predictions. However, while increasingly on the rise its potential is still widely underused in conservation, (spatial) ecological applications and for inference. Our results show that it informs ecological and biogeographical theories as well as being suitable for conservation applications, specifically when the study area is undersampled. This method helps to save model-selection time and effort, and it allows robust and rapid assessments 
and decisions for efficient conservation. 
1 Why choose Random Forest to predict rare species distribution with few samples in large

2 undersampled areas? Three Asian crane species models provide supporting evidence

$4{ }^{1}$ College of Nature Conservation, Beijing Forestry University, P.O. Box 159, Beijing 100083,

5 China

$6{ }^{2}$ Key Laboratory of Water Cycle and Related Land Surface Processes, Institute of Geographic

7 Sciences and Natural Resources Research, University of Chinese Academy of Sciences, Beijing

8 100101, China

$9{ }^{3}$ EWHALE Lab, Department of Biology and Wildlife, Institute of Arctic Biology, University of 10 Alaska Fairbanks (UAF), 419 Irving I, P.O. Box 757000, AK 99775, USA

11 Corresponding author:

12 Yumin Guo

13 College of Nature Conservation, Beijing Forestry University, P.O. Box 159, Beijing 100083, 14 China

15 guoyumin@bjfu.edu.cn 


\section{ABSTRACT}

17 Species distribution models (SDMs) have become an essential tool in ecology, biogeography,

18 evolution, and more recently, in conservation biology. How to generalize species distributions in

19 large undersampled areas, especially with few samples, is a fundamental issue of SDMs, and

20 beyond. In order to explore this issue, we used the best available presence records for the Hooded

21 Crane (Grus monacha, n=33), White-naped Crane (Grus vipio, n=40), and Black-necked Crane

22 (Grus nigricollis, $\mathrm{n}=75$ ) in China as three case studies, employing four powerful and commonly

23 used machine learning algorithms to map the breeding distributions of the three species: TreeNet

24 (Stochastic Gradient Boosting, Boosted Regression Tree Model), Random Forest, CART

25 (Classification and Regression Tree) and Maxent (Maximum Entropy Models) In addition we

26 developed an ensemble forecast by averaging predicted relative indeces of occurrence for the four

27 models results. Commonly-used model performance metrics (Area under ROC (AUC) and true

28 skill statistic (TSS)) were employed to evaluate model accuracy. Latest satellite tracking data and

compiled literature data were used as two independent testing datasets to confront model

predictions. We found Random Forest demonstrated the best performance for most assessment

methods, provided a better model fit to the testing data, and achieved better species range maps

for each crane species in undersampled areas. Random Forest has been generally available for more than 20 years, and by now, has been known to perform extremely well in ecological predictions. However, while increasingly on the rise its potential is still widely underused in conservation, in (spatial) ecological applications and for inference. Our results show that it informs ecological and biogeographical theories as well as being suitable for conservation applications, specifically when the study area is undersampled. This method helps to save modelselection time and effort, and it allows robust and rapid assessments and decisions for efficient conservation. 
40 INTRODUCTION

41 Species distribution models (SDMs) are empirical ecological models that relate species

42 observations to environmental predictors (Guisan \& Zimmermann, 2000; Drew et al., 2011).

43 SDMs have become an increasingly important and now essential tool in ecology, biogeography,

44 evolution and, more recently, in conservation biology (Guisan et al., 2013), management

45 (Cushman \& Huettmann, 2010), impact assessments (Humphries \& Huettmann, 2014) and

46 climate change research (Lei et al., 2011; Mi et al., 2016). To generalize and infer from a model,

47 or model transferability is defined as geographical or temporal cross-applicability of models

48 (Thomas \& Bovee 1993; Kleyer 2002; Randin et al., 2006). It is one important feature in SDMs,

49 a base-requirement in several ecological and conservation biological applications (Heikkinen et

50 al., 2012). In this study, we used generality (transferability) as the concept of generalizing

51 distribution from sampled areas to unsampled areas (extrapolation beyond the data) in one study

52 area.

53 Detailed distribution data for rare species in large areas are rarely available in SDMs (Pearson

54 et al., 2007; Booms et al., 2010). However, they are among the most needed for their

55 conservation to be effective. Collecting and assembling distribution data for species, especially

56 for rare or endangered species in remote wilderness areas is often a very difficult task, requiring a

57 large amount of human, time and funding sources (Gwena et al., 2010; Ohse et al., 2009).

58 Recent studies have suggested that machine-learning (ML) methodology, may perform better

59 than the traditional regression-based algorithms (Elith et al., 2006). TreeNet (boosting; Friedman

60 2002), Random Forest (bagging; Breiman, 2001), CART (Breiman et al., 1984) and Maxent

61 (Phillips et al., 2004) are considered to be among the most powerful machine learning algorithms

62 and for common usages (Elith et al., 2006; Wisz et al., 2008; Williams et al., 2009; Lei et al., 
63 2011) and for obtaining powerful ensemble models (Araújo and New 2007; Hardy et al., 2011).

64 Although Heikkinen et al. (2012) compared the four SDMs techniques' transferability in their

65 study, they did not test with rare species and few samples in undersampled areas. It is important

66 to understand that the software platform of the former three algorithms (Boosted Regression

67 Trees, Random Forest and CART) applied by Heikkinen et al. (2012) from the R software

68 ("BIOMOD" framewok) comes without a GUI and lacks sophisticated optimization, sample

69 balancing and fine-tuning, but as they are commonly used though by numerous SDM modelers.

70 Instead, we here run these models in the Salford Predictive Modeler (SPM version 7) by Salford

71 Systems Ltd (https://www.salford-systems.com/). These algorithms in SPM are further optimized

72 and improved by one of the algorithm's original co-authors (especially for TreeNet and Random

73 Forest). It runs with a convenient GUI, and produces a number of descriptive results and graphics

74 which are virtually not available in the $\mathrm{R}$ version. While this is a commercial software, it is

75 usually available on a 30 days trial version (which suffices for most model runs we know. As

76 well, some of the features of the randomForest $\mathrm{R}$ package, most notably the ability to produce

77 partial dependence plots (Herrick 2013), are not directly implemented yet in SPM7 (but they can

78 essentially be obtained by running TreeNet in a Random Forest model).

79 Model generality (transferability) testing could offer particularly powerful for model evaluation (Randin et al., 2006). Independent observations from a training data set has been

81 recommended as a more proper evaluations of models (Fielding \& Bell 1997; Guisan and Zimmermann 2000). So the use of an independent geographically (Fielding \& Haworth, 1995) or temporally (Boyce et al., 2002; Araujo et al., 2005b) testing data set is encouraged to assess the generality of different SDMs techniques. Data from museum specimen, published literature (Graham et al., 2004) as well as tracking are good source to assess model generality (transferability) performance. In addition, how the distribution map links with reality data, 
88 employed as a metric to assess model performance and generalization. Arguably, if model

89 predictions perform very well there, great progress is provided and usually done cost-effective 90 too. Whereas, predictions on existing knowledge and data offers less progress. The model

91 prediction and conservation frontier obviously sits in the unknown and to provide progress there

92 (Breiman 2001, Drew et al. 2011).

93 In this study, we investigated models for the best-available data for three species in East Asia 94 as test cases: Hooded Cranes (Grus monacha, $\mathrm{n}=33$ ), White-naped Cranes (Grus vipio, $\mathrm{n}=40$ ) and 95 Black-necked Cranes (Grus nigricollis, n=75). Four machine-learning model algorithms 96 (TreeNet, Random Forest, CART and Maxent) were applied to map breeding distributions for 97 these three crane species which otherwise lack empirically derived distribution information. In 98 addition, two kinds of independent testing data sets (latest satellite tracking data, and compiled 99 literature data (Threatened Birds of Asia: Collar et al., 2001) were obtained to test the 100 transferability of the four model algorithms. The purpose of this investigation is to explore 101 whether there is a SDM technique among the four algorithms that could generate reliable and 102 accurate distributions with high generality for rare species using few samples but in large undersampled areas? Results from this research could be useful for the detection of rare species and enhance fieldwork sampling in large undersampled areas which would save money and effort, as well as advance the conservation management of those species.

\section{MATERIALS AND METHODS}

107

\section{Species data}

In our 13 combined years of field work, we have collected 33 Hooded Crane nests (2002-2014), 40 White-naped Crane nests (2009-2014), and 75 Black-necked Crane nests (2014) (see Fig. 1), during breeding seasons. We used these field samples (nests) to represent species presence points referenced in time and space. 


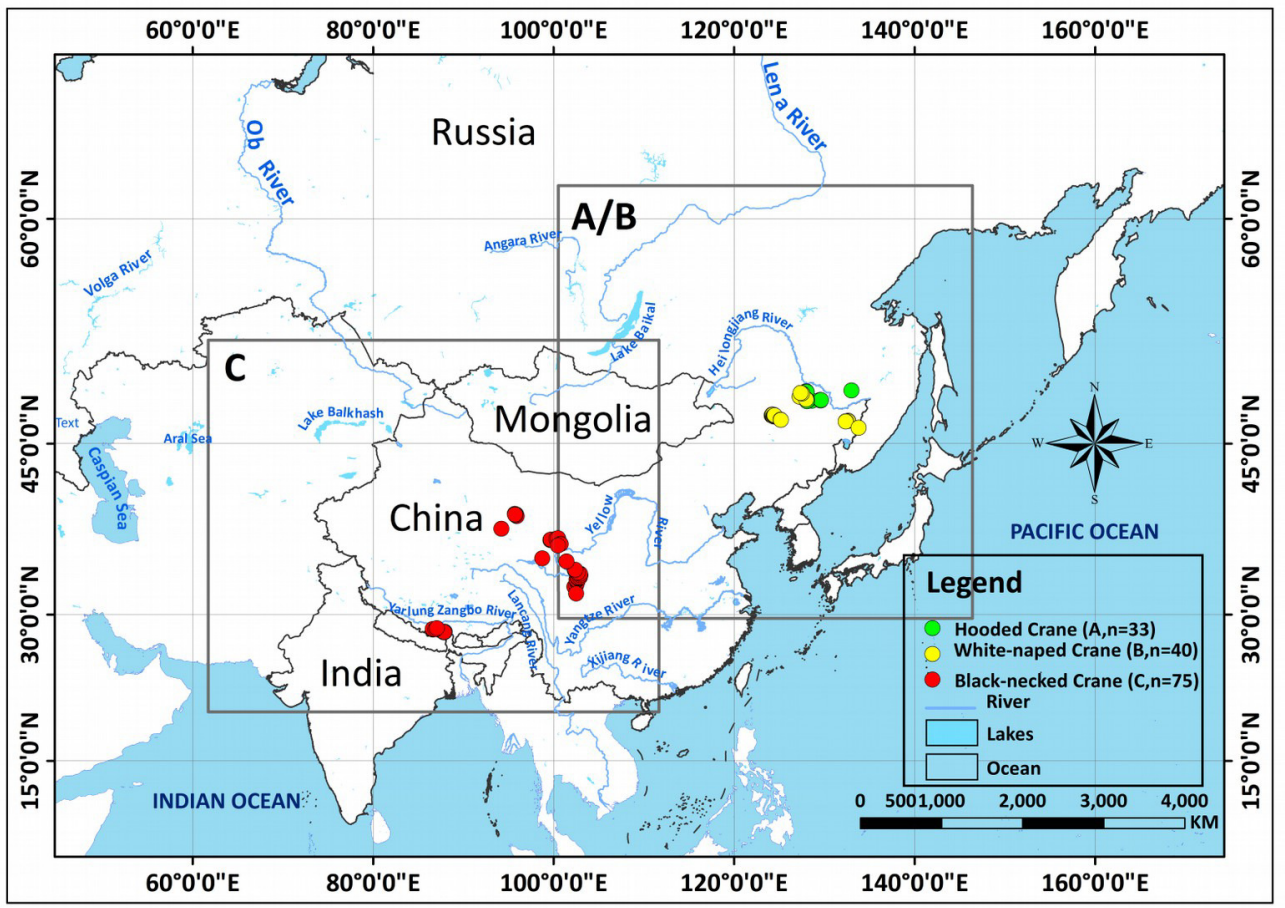

Figure 1 Study areas for three species cranes.

\section{Environmental variables}

We used 21 environmental layers at a 30-s spatial resolution in a GIS format and that were known to correlate with bird distribution and as proxies of habitats predictors. They included bioclimatic factors (bio_1-7, bio_12-15), topographical factors (altitude, slope, and aspect), water factors (distance to river, distance to lake, and distance to coastline), inference factors (distance to road, distance to rail road, and distance to settlements), and land cover factors (for detailed information, see Table 1). Most of these predictors were obtained from open access sources. Bioclimate factors were obtained from the WorldClim database (http://www.worldclim.org), while aspect and slope layer were derived from the altitude layer in ArcGIS, which was also initially obtained from the WorldClim database. Road, railroad, river, lake and coastline and settlement 
126 variables and 10 other environmental variables, and then reduced predictors by $\mathrm{AIC}, \mathrm{BIC}$,

127 varclust, PCA and FA analysis. When we compared the distribution maps overlaying with

128 independent data set generated by Random Forest model, we found the model based on 21

129 predictors have the best performance for Hooded Cranes, and the best level for White-naped

130 Crane and Black-necked Cranes (see Supplement S1). Therefore, we decided to constructed

131 models with 21 predictors for the all three cranes and four machine-learning techniques. All

132 spatial layers of these environmental variables were resampled in ArcGIS to a resolution of 30-s

133 to correspond to that of the bioclimatic variables and for a meaningful high-resolution

134 management scale.

Put Table 1 here

136 Table 1 Environmental GIS layers used to predict breeding distributions of three cranes.

\begin{tabular}{|c|c|c|c|}
\hline $\begin{array}{l}\text { Environmental } \\
\text { Layers } \\
\end{array}$ & Description & Source & Website \\
\hline Bio_1 & $\begin{array}{l}\text { Annual mean Temperature ( } \\
{ }^{\circ} \mathrm{C} \text { ) }\end{array}$ & WorldClim & http://www.worldclim.org/ \\
\hline Bio_2 & $\begin{array}{l}\text { Monthly mean (max temp - } \\
\text { min temp) } \\
\left({ }^{\circ} \mathrm{C}\right)\end{array}$ & WorldClim & http://www.worldclim.org/ \\
\hline Bio_3 & $\begin{array}{l}\text { Isothermality }(\mathrm{BIO} 2 / \mathrm{BIO} 7) \\
\left(* 100{ }^{\circ} \mathrm{C}\right)\end{array}$ & WorldClim & http://www.worldclim.org/ \\
\hline Bio_4 & $\begin{array}{l}\text { Temperature seasonality } \\
\text { (standard deviation } * 100 \\
{ }^{\circ} \mathrm{C} \text { ) }\end{array}$ & WorldClim & http://www.worldclim.org/ \\
\hline Bio_5 & Max temperature of & WorldClim & http://www.worldclim.org/ \\
\hline Bio_6 & $\begin{array}{l}\text { warmest month }\left({ }^{\circ} \mathrm{C}\right) \\
\text { Min temperature of Coldest }\end{array}$ & WorldClim & http://www.worldclim.org/ \\
\hline Bio_7 & $\begin{array}{l}\text { month }\left({ }^{\circ} \mathrm{C}\right) \\
\text { Annual temperature range } \\
\left(\text { BIO5-BIO6) }\left({ }^{\circ} \mathrm{C}\right)\right.\end{array}$ & WorldClim & http://www.worldclim.org/ \\
\hline Bio_12 & Annual precipitation (mm) & WorldClim & http://www.worldclim.org/ \\
\hline Bio_13 & Precipitation of wettest & WorldClim & http://www.worldclim.org/ \\
\hline Bio_14 & $\begin{array}{l}\text { month }(\mathrm{mm}) \\
\text { Precipitation of driest }\end{array}$ & WorldClim & http://www.worldclim.org/ \\
\hline
\end{tabular}




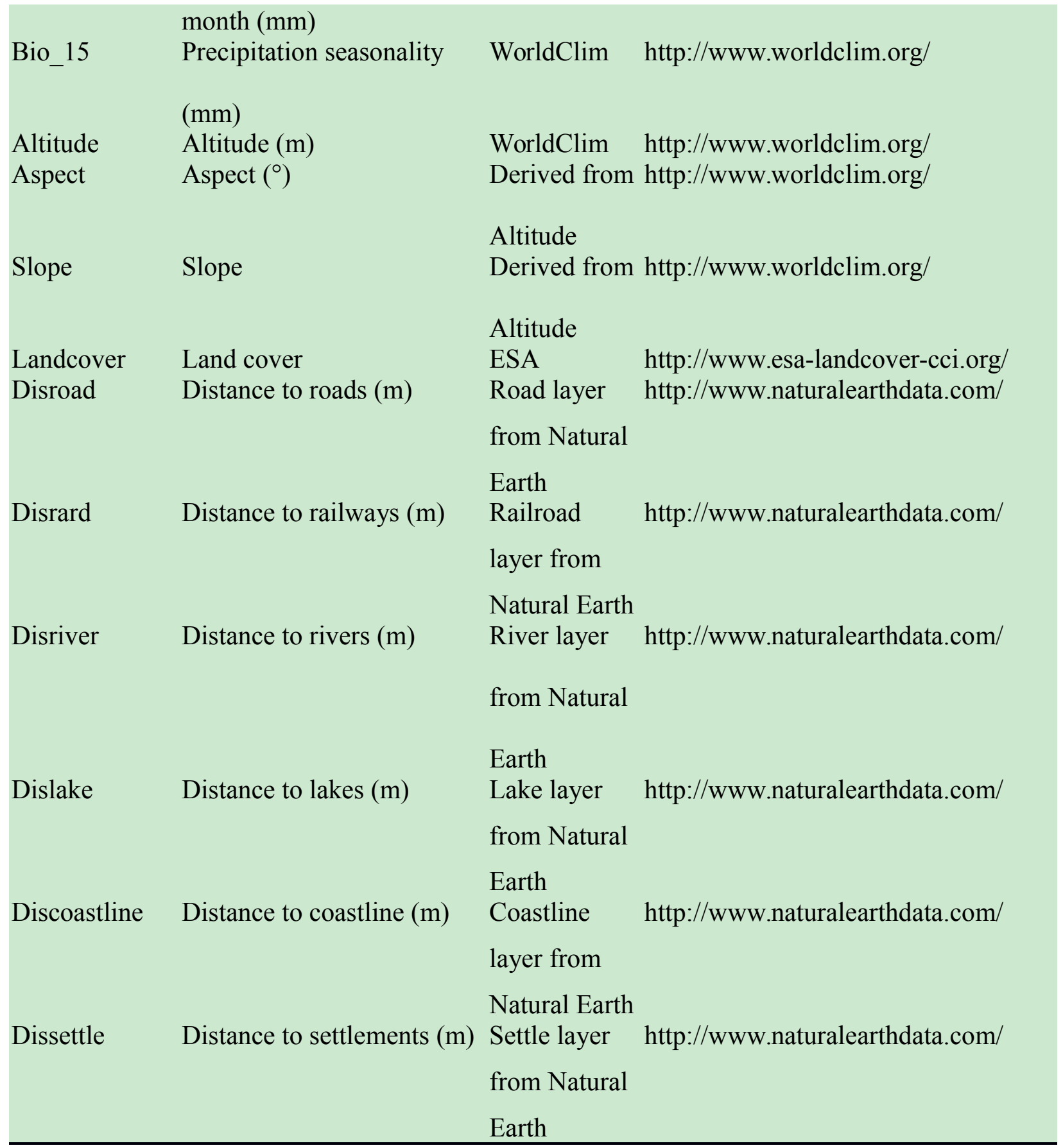

\section{Model development}

We created TreeNet, Random Forest, CART, Maxent models and ensemble models (averaged value of the former four model results) for Hooded Cranes, White-naped Cranes and Black-naped Cranes. These four model algorithms are considered to be among the best performing machine 141 learning methods (more information about these four models can be seen in the references by 142 Breiman et al., 1984, Breiman 2001, Friedman 2002, Phillips et al., 2004, Hegel et al., 2010). The 
143 first three machine learning models are binary (presence-pseudo absence) models and were 144 handled in Salford Predictive Modeler 7.0 (SPM). For more details on TreeNet, Random Forest 145 and CART in SPM and their performances, we refer readers to the user guide document online 146 (https://www.salford-systems.com/products/spm/userguide). Several implementations of these 147 algorithms exist. Approximately 10,000 'pseudo-absence' locations were selected by random 148 sampling across the study area for each species using the freely available Geospatial Modeling 149 Environment (GME; Hawth’s Tools; Beyer 2013; see Booms et al., 2010 and Ohse et al., 2009 for examples). We extracted the habitat information from the environmental layers for presence and pseudo-absence points for each crane, and then constructed models in SPM with these data. In 152 addition, we used balanced class weights, and 1000 trees were built for all models to find an optimum within, others used default settings.

154 For the predictions, we created a 'lattice' (equally spaced points across the study area; approximately $5 \times 5 \mathrm{~km}$ spacing for the study area). For the lattice, we extracted information from the same environmental layers (Table 1) as described above for each point and then used the model to predict ('score') bird presence for each of the regular lattice points. For visualization, we imported the dataset of spatially referenced predictions ('score file') into GIS as a raster file and interpolated for visual purposes between the regular points using inverse distance weighting (IDW) to obtain a smoothed predictive map of all pixels for the breeding distributions of the three cranes (as performed in Ohse et al., 2009 and Booms et al., 2010). The fourth algorithm we employed, Maxent, is commonly referred to as a presence-only model; we used Maxent 3.3.3k (it can be downloaded for free from http://www.cs.princeton.edu/ schapire/maxent/) to construct our models. To run Maxent, we followed the 3.3.3e tutorial for ArcGIS 10 (Young et al., 2011) and used default settings. 


\section{Testing data and model assessment}

167 We applied two types of testing data in this study: one consisted of satellite tracking data, and

168 the other was represented by data from the literature. Satellite tracking data were obtained from 4

169 individual Hooded Cranes and 8 White-naped Cranes that were tracked in the breeding regions at

170 stopover sites (for more details regarding the information for tracked cranes, please see

171 Supplement S2). The satellite tracking devices could provide 24 data points per day (Databases

172 could be available upon request). Here, we chose points that had a speed of less than $5 \mathrm{~km} / \mathrm{h}$

173 during the period from $1^{\text {st }}$ May to $31^{\text {th }}$ June for Hooded Cranes and $15^{\text {th }}$ April to $15^{\text {th }}$ June for

174 White-naped Cranes as the locations of the breeding grounds for these two cranes. The total

175 numbers of tracking data points were 4,963 and 7,712 (Hooded Cranes and White-naped Crane,

176 respectively. We didn't track Black-necked Cranes, so there was no tracking testing data for this

177 species). The literature data for this study were obtained by geo-referencing the location points of 178 detections from 1980-2000 (ArcGIS 10.1) from Threatened Birds of Asia: the BirdLife

179 International Red Data Book (Collar et al., 2001). From this hardcopy data source, we were able 180 to obtain and digitize 27 breeding records for Hooded Cranes, 43 breeding records for White-

naped Cranes, and 53 breeding records for Black-necked Cranes (see Fig. 2a, 2b, 2c). Here we digitized the only available crane data for these three species in East-Asia into a database.

In addition, we generated 3,000 random points for Hooded Cranes and White-naped Cranes, and 5,000 random points for Black-necked Cranes as testing pseudo-absence points in their respective study areas. And then, the literature locations (additional presence points for testing) and random points location (testing absence points) that contrasted with the associated predictive value of RIO extracted from the relative prediction map, which were used to calculate receiver operating characteristic (ROC) curves and the true skill statistic (TSS) (Hijmans and Graham, 2006). The area under the ROC curve (AUC) is commonly used to evaluate models in species 
190 distributional modeling (Manel et al., 2001, McPherson et al., 2004). TSS was also used to 191 evaluate model performance; we used TSS because it has been increasingly applied as a simple 192 but robust and intuitive measure of the performance of species distribution models (Allouche et 193 al., 2006).

Put Fig.2 here 

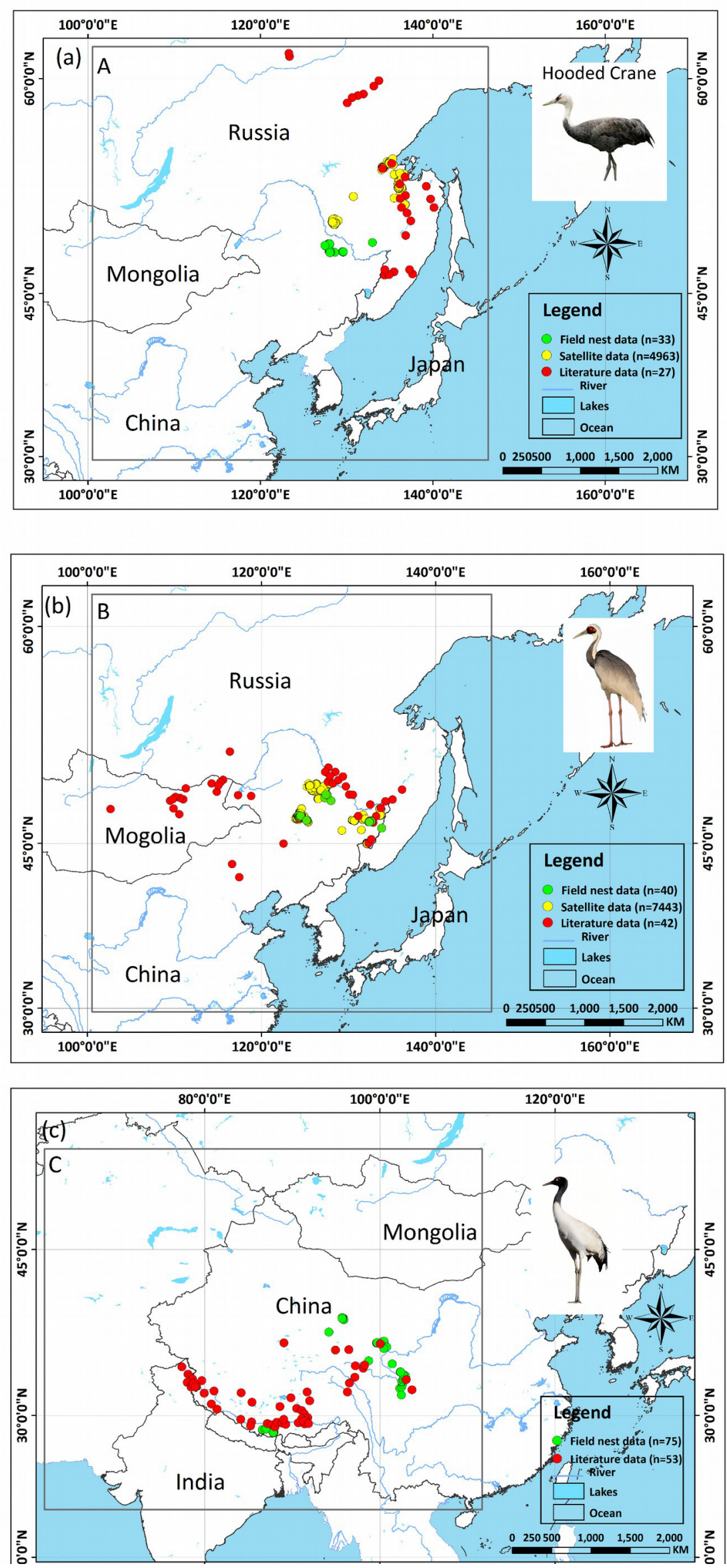
195 Figure 2 Detailed study areas showing the presence of and testing data used for the three cranes.

196 2a) Hooded Cranes, 2b) White-naped Cranes, 2c) Black-necked Cranes. 
197 To assess models transferability, we extracted the predictive value of the relative index of 198 occurrence (RIO) for testing data sets from the prediction maps using GME. We then constructed 199 resulting violin plots in $\mathrm{R}$ for these extracted RIOs to visualize their one-dimensional distribution. 200 This method allowed us to examine the degree of generalizability based on the local area with 201 samples to predict into undersampled areas that are otherwise unsampled in the model 202 development (=areas without training data). In addition, AUC is also commonly used to assess 203 model transferability in our study referring Randin et al. (2006).

\section{RESULTS}

\section{Model performance}

The results for AUC and TSS, two metrics commonly used to evaluate model accuracy, are 207 listed in Table 2. For the four SDMs technique, our results showed that the AUC values for Random Forest were always highest $(>0.625)$, ranking this model in first place, followed by Maxent $(>0.558)$, and then either CART or TreeNet $(>=0.500)$. TSS showed us consistent results, as was the case for AUC, and Random Forest performed the best $(>0.250)$ followed by Maxent $(>0.137)$ for all three crane species, CART took the third place for Black-necked Cranes, and TreeNet performed better than CART for White-naped Cranes. And the results showed there was a trend that the value of these three metrics increased with an increase of nest site samples (33 to 75, Hooded Crane to Black-necked Crane, see Table. 2). Comparing the results of Random Forest with ensemble models, we found their performance were close. Random Forest obtained better models for Hooded Cranes and White-naped Cranes cases, the ensemble model performed better for Black-necked Cranes.

Table 2 AUC and TSS values for four machine learning models and their ensemble model with three crane species based on literature testing data. 


\begin{tabular}{|c|c|c|c|c|c|}
\hline \multirow{3}{*}{$\begin{array}{l}\text { Accuracy metric } \\
\text { (samples) }\end{array}$} & \multicolumn{5}{|c|}{ Species distribution model } \\
\hline & TreeNet & Random & CART & Maxent & Ensemble \\
\hline & \multicolumn{5}{|c|}{ Forest } \\
\hline \multicolumn{6}{|c|}{ Hooded Crane (Grus monacha, $\mathrm{n}=33$ sites) } \\
\hline AUC & 0.504 & 0.625 & 0.500 & 0.558 & 0.558 \\
\hline TSS & 0.000 & 0.250 & 0.000 & 0.137 & 0.117 \\
\hline \multicolumn{6}{|c|}{ White-naped Crane (Grus vipio, $\mathrm{n}=40$ sites) } \\
\hline AUC & 0.605 & 0.754 & 0.564 & 0.712 & 0.754 \\
\hline TSS & 0.210 & 0.509 & 0.128 & 0.424 & 0.508 \\
\hline \multicolumn{6}{|c|}{ Black-necked Crane (Grus nigricollis, $\mathrm{n}=75$ sites) } \\
\hline AUC & 0.528 & 0.830 & 0.672 & 0.805 & 0.843 \\
\hline TSS & 0.055 & 0.660 & 0.345 & 0.611 & 0.686 \\
\hline
\end{tabular}

\section{Model generalization} Hooded Crane models (Fig. 3a), the RIO for most satellite tracking data indicated that TreeNet, and CART predicted with a value around 0; Ensemble model demonstrated a slightly higher value than the other three models but was still much lower than Random Forest. Fig. 3b indicates the same situation than found in Fig. 3a: Random Forest still performed better than the other three models (median values in Random Forests were close to 1.00). TreeNet had a median RIO value of approximately 0.71 , followed by Maxent (median was 0.37 ) and then ensemble and CART. While some tracking points had a low RIO value in TreeNet, the majority of RIO values for CART remained in the 0.20 range. 


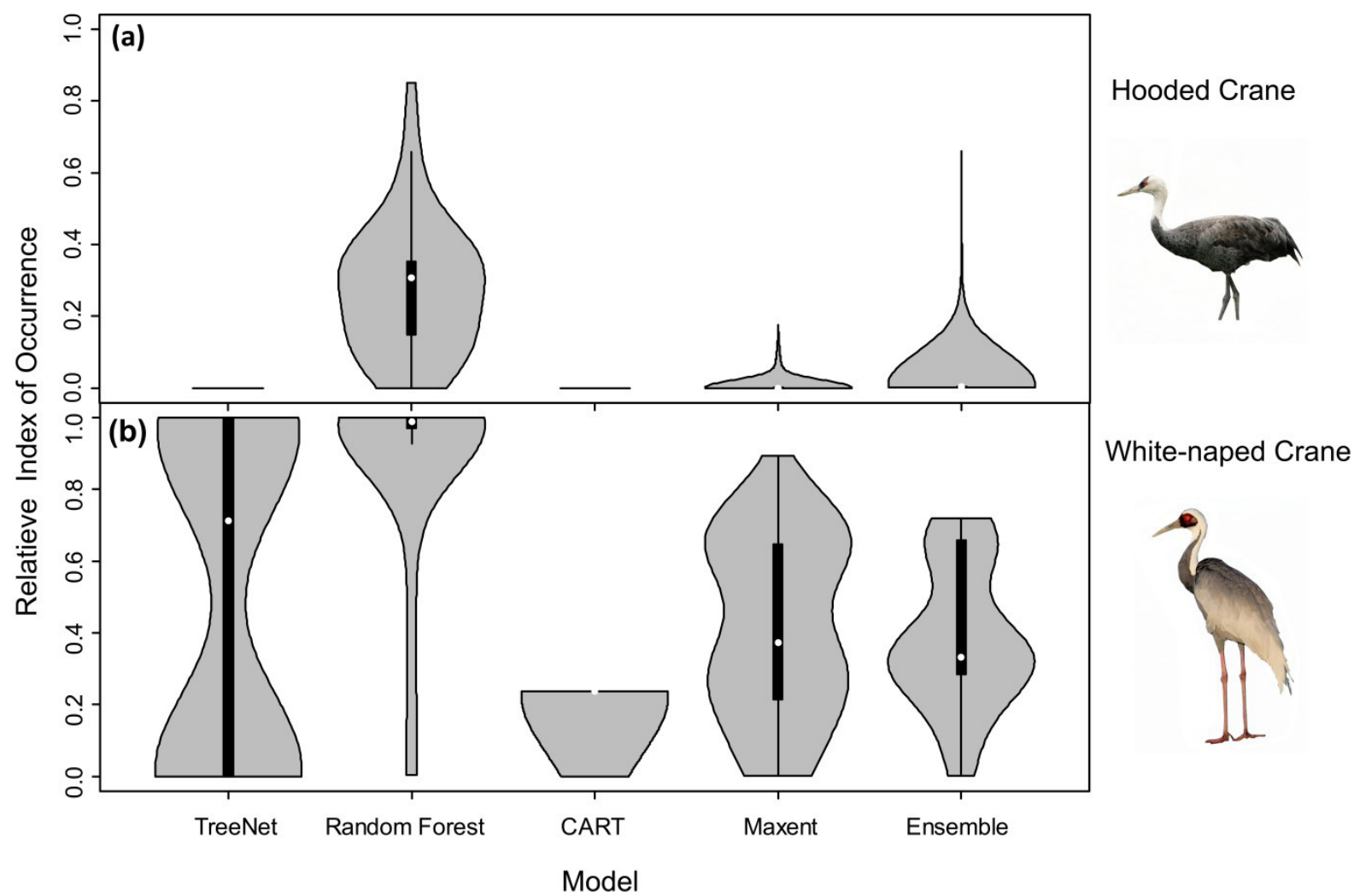

Figure 3 Violin plots of the Relative Index of Occurrence (RIO) for four SDMs and ensemble model for Hooded Cranes and White-naped Cranes based on satellite tracking data. 3a) violin plots of Hooded Cranes, 3b) violin plots of White-naped Cranes.

Violin plots of the RIOs values for the three cranes extracted for the literature data from the prediction maps (Fig. 4) demonstrated consistent trends (Fig. 3), indicating that Random Forest performed best across all models of the three species. In Fig. 4a, the RIO values for Random Forest ranged from 0 to 0.48 , and most RIO values were below 0.1 ; the RIO values for the other three SDMs method were 0, the ensemble model performed a little bit better. As showed in Fig. $4 \mathrm{~b}$, most RIO values for Random Forest were below 0.7 , and the median value was approximately 0.20 , followed by Maxent and then CART. The violin plots for Black-necked Cranes (Fig. 4c) indicated that TreeNet performed the worst, although there were some pixels that had high RIO values, followed by the ensemble model and then Maxent. The best performer was still Random Forest, and its RIOs were distributed evenly to a certain extent with a median value 
246 of 0.44 . The results of AUC, as mentioned in "Model performance" part (Table 2), showed 247 consistent results with violin plots, Random Forest always get the highest value and has the best 248 generalization.

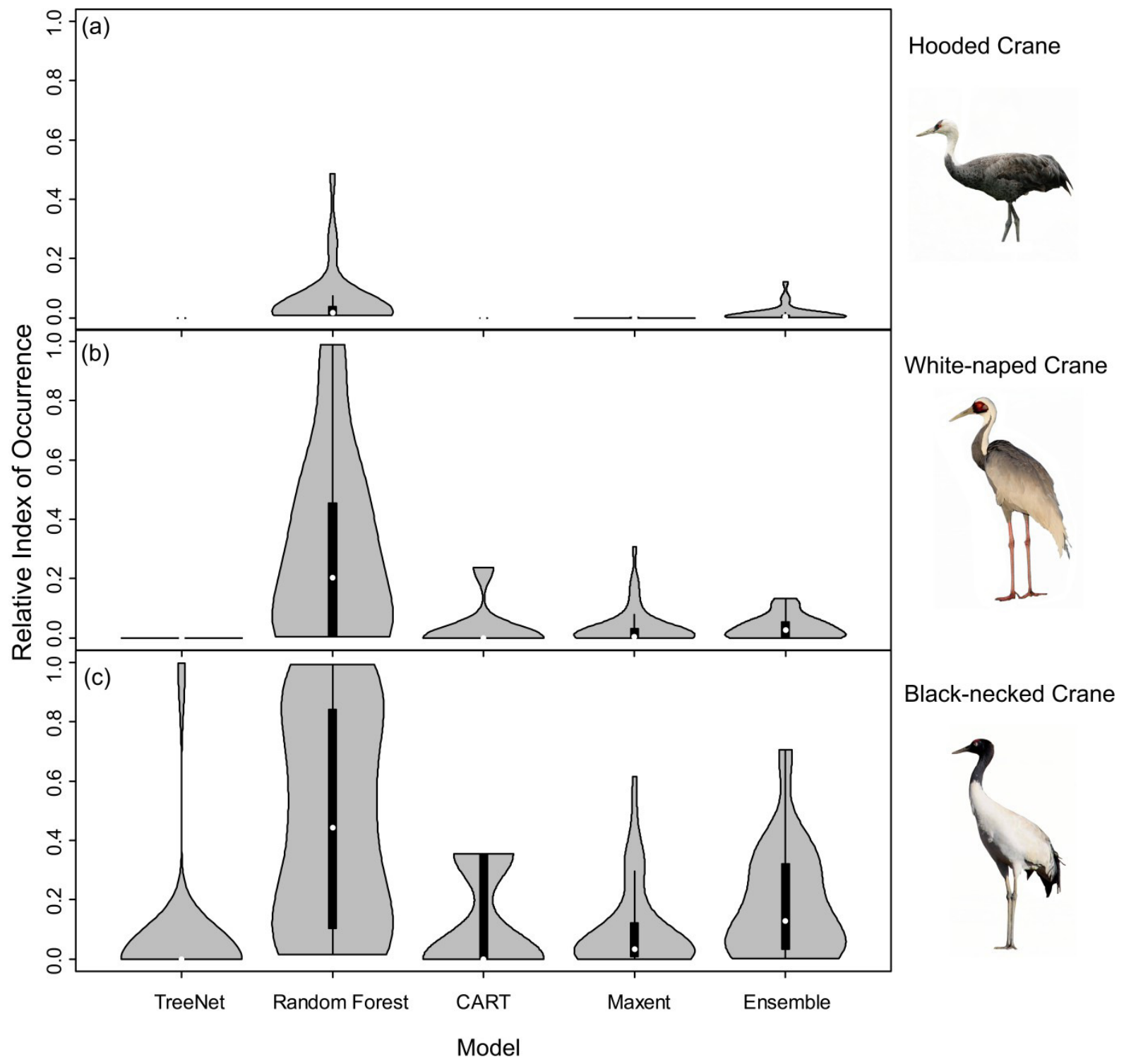

Figure 4 Violin plots of Relative Index of Occurrence (RIO) values for four SDMs and ensemble model for three cranes based on calibration data from Threatened Birds of Asia. 4a) Violin plots for Hooded Cranes, 4b) violin plots for White-naped Cranes, 4c) violin plots for Black-necked Cranes. 


\section{Spatial assessment using a testing data overlay prediction map}

An assessment of niche prediction beyond the local area where samples were located represents a real test of the generalizability of the model predictions in undersampled areas. This approach was used to evaluate whether testing data (satellite tracking data and literature data) locations matched predictions of the potential distribution area, as a spatial assessment of model performance. It's a spatial and visual method to show the transferability of SDMs from sampled to unsampled areas. From the results (Fig.s 5, 6 and 7. Digital version for each subgraph could be available request), we found that Random Forest demonstrated the strongest performance to handle generality (transferability), and a high fraction of testing data locations were predicted in the distribution areas of the three cranes (Fig.s 5b, 5g, 6b, 6g, 7b, 7g). The order of the generality of the remaining four models was: ensemble model followed by Maxent, CART and then TreeNet. Note, however, that the capacities of these models to predict well in undersampled areas were weaker than Random Forest, it holds particularly for areas that were further away from the sample areas (Fig.s 5, 6 and 7). In addition, we found that the generality increased with sample size (33 to 75, Hooded Crane to Black-necked Crane, see Fig.s 5, 6 and 7). This means, as expected and known, that a higher sample size makes models more robust and better to generalize from.

\section{Put Fig. 5 Here}



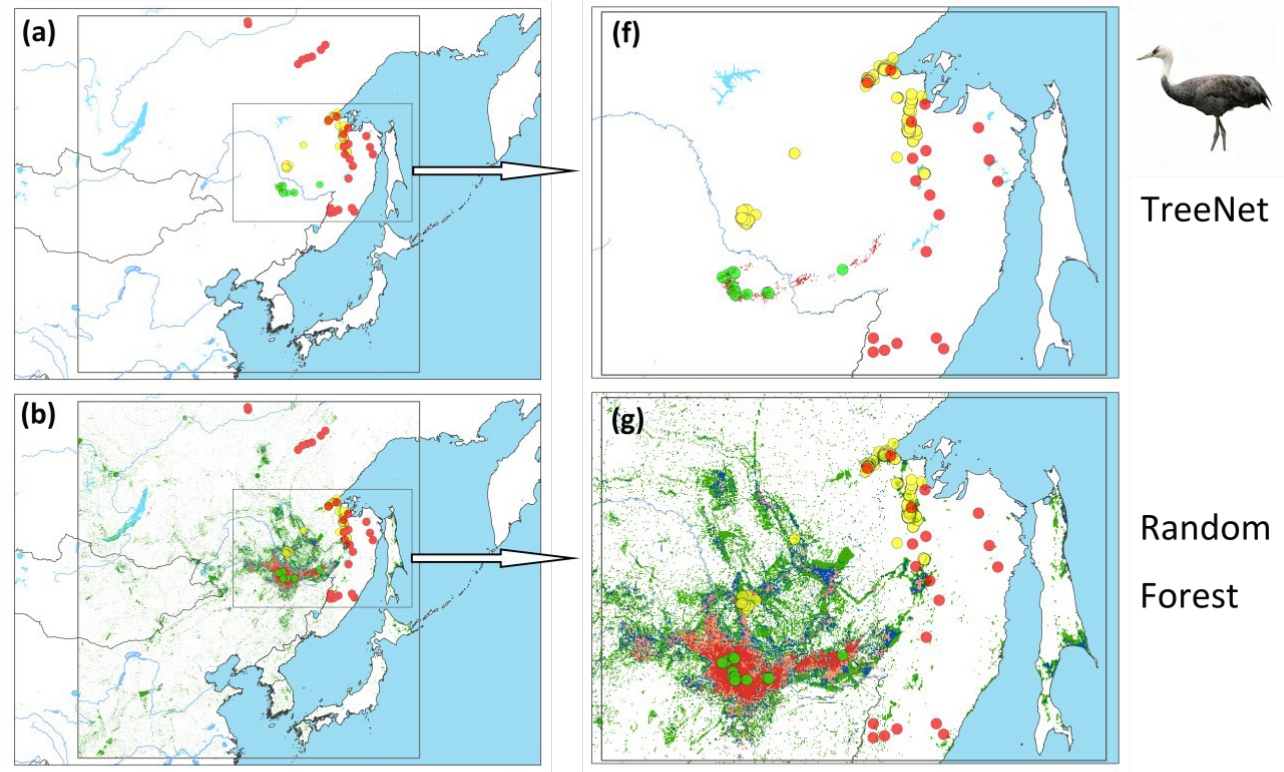

\section{Random \\ Forest}
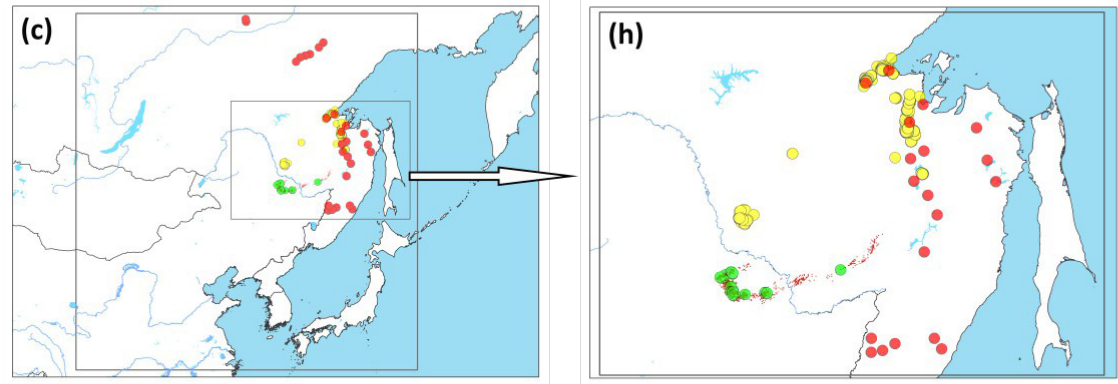

CART
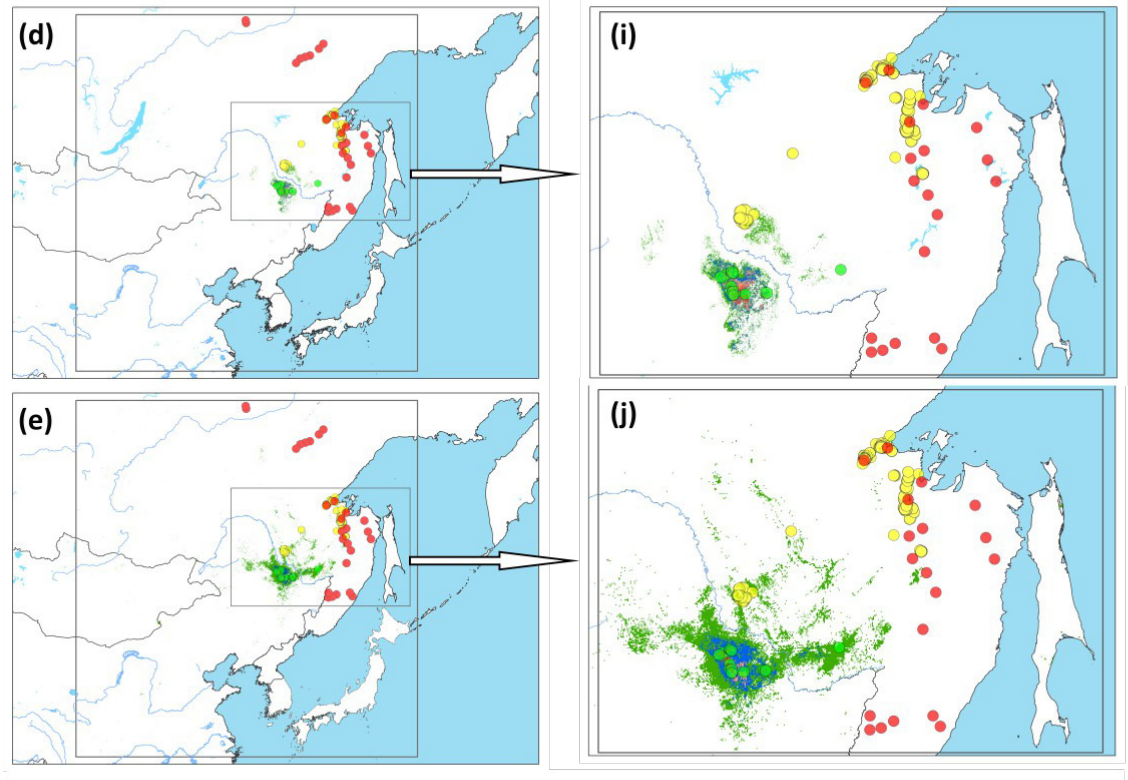

Ensemble

Field nest data ( $n=33) \bigcirc$ Satellite data $(n=4963)$

- Literature data ( $\mathrm{n}=\mathbf{2 7}$ )

$0.00-0.20$

$0.20-0.40$

$0.40-0.60$

$0.60-0.80$

$0.80-1.00$

272 Figure 5 Prediction maps for Hooded Cranes and zoomed-in maps showing the four models (TreeNet,

273 Random Forest, CART and Maxent) and ensemble model in detail. 5a-5e) prediction map for Hooded 
Cranes, $5 f-5 j$ ) zoomed-in map for Hooded Cranes.
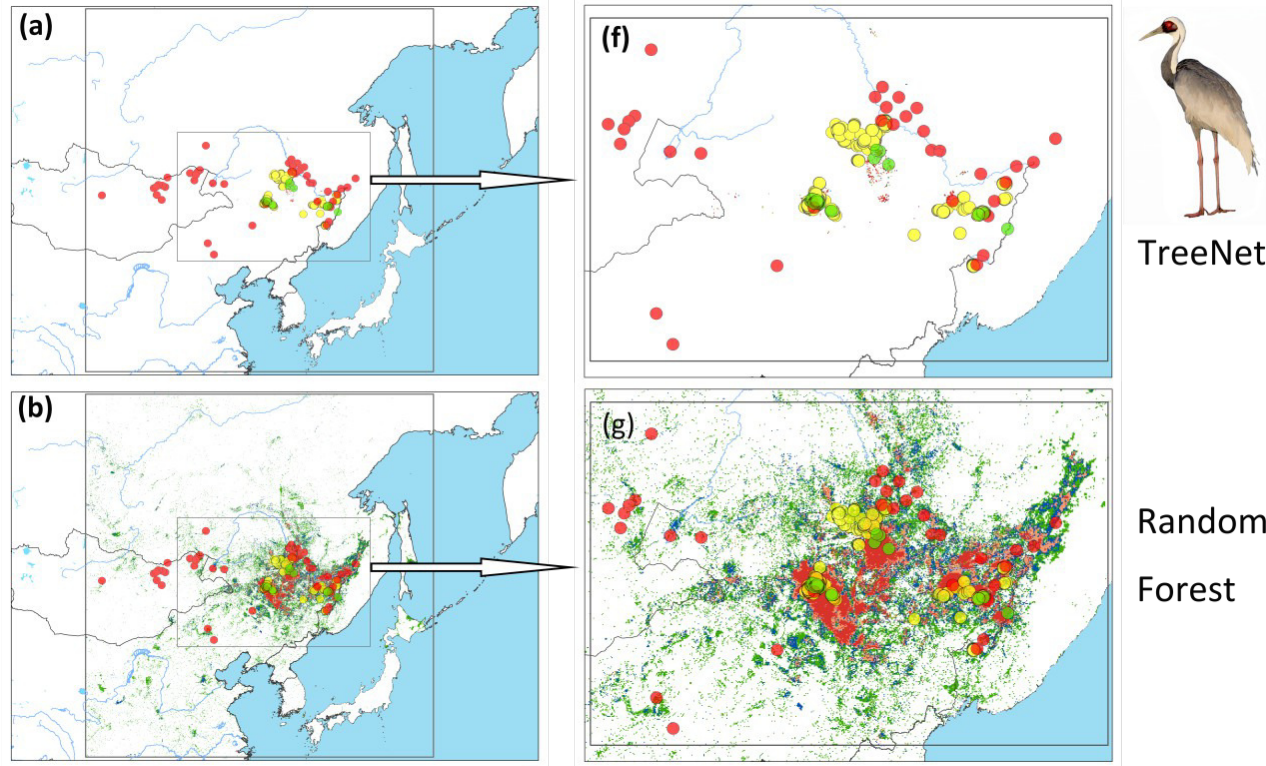

TreeNet
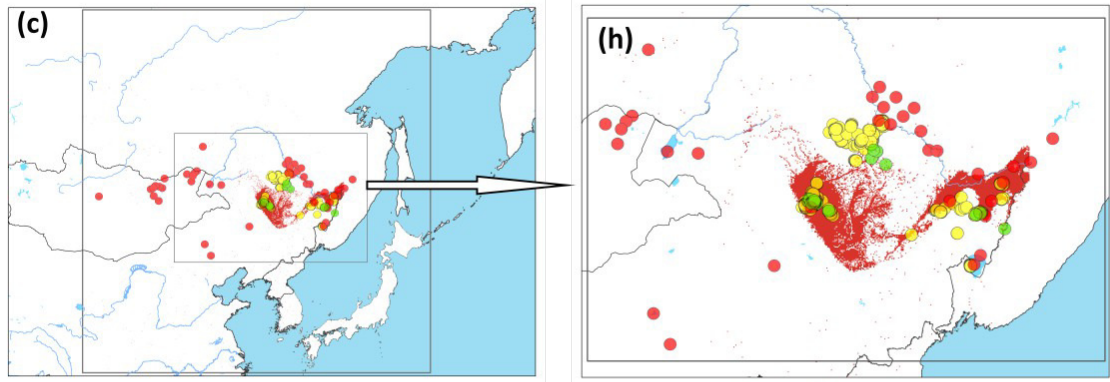

\section{Random \\ Forest}
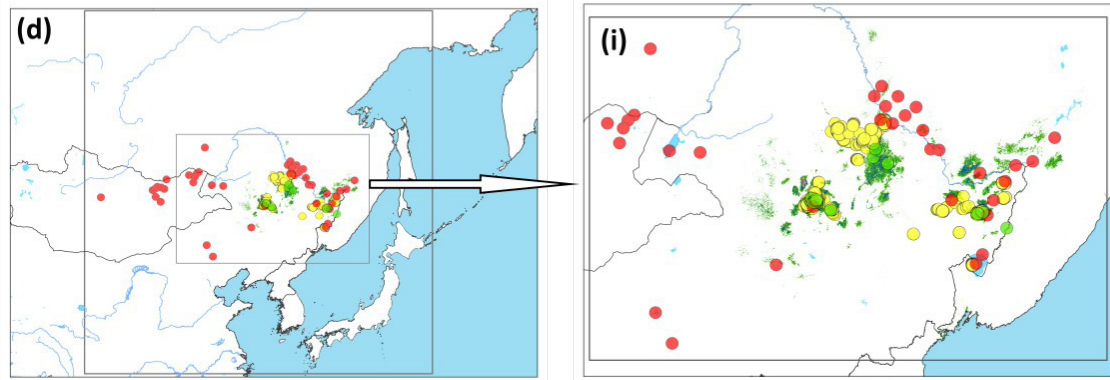

CART
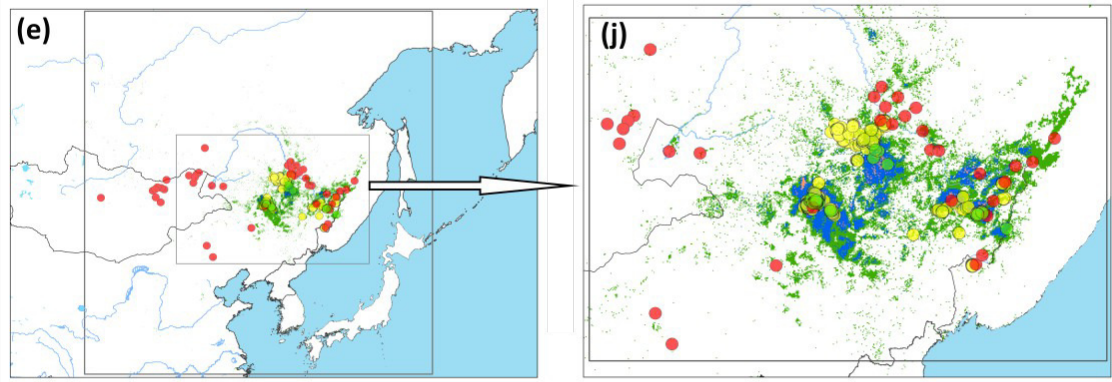

Maxent

Field nest data $(n=40) \quad$ Satellite data $(n=7712)$

- Literature data $(n=43)$

$\square 0.00-0.20 \square 0.20-0.40$

$0.40-0.60$

$0.60-0.80$

$0.80-1.00$

Ensemble

275 Figure 6 Prediction maps for White-naped Cranes and zoomed-in maps showing the four models (TreeNet, 
276 Random Forest, CART and Maxent) and ensemble model in detail. 6a-6e) prediction map for White-naped 277 Cranes, 6f-6j) zoomed-in map for White-naped Cranes.

278

Put Fig. 6 Here 

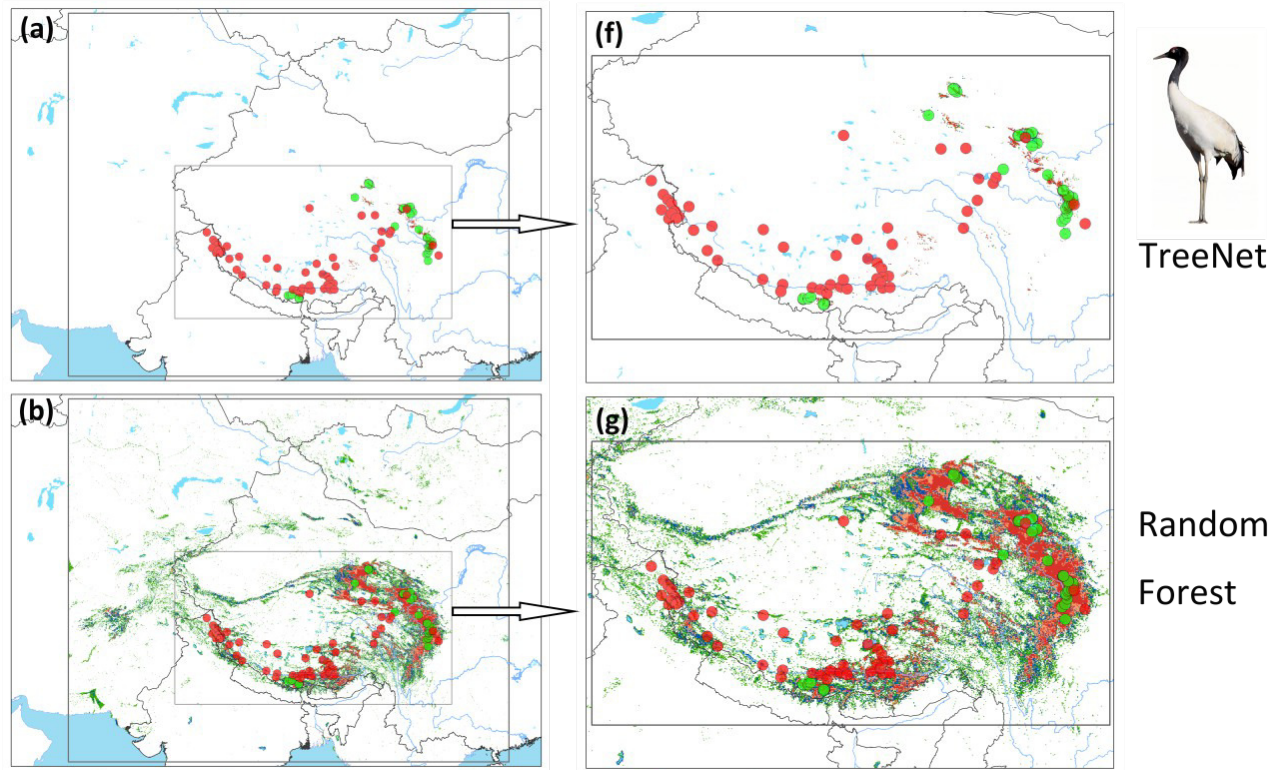

Random
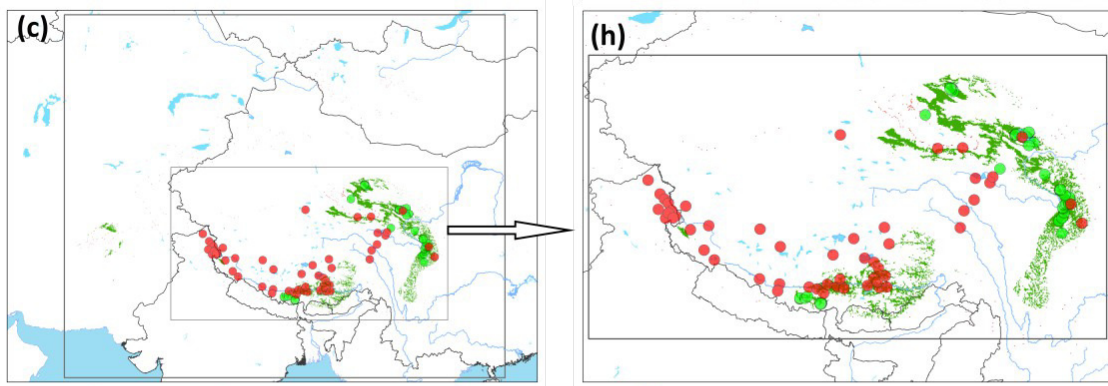

Forest
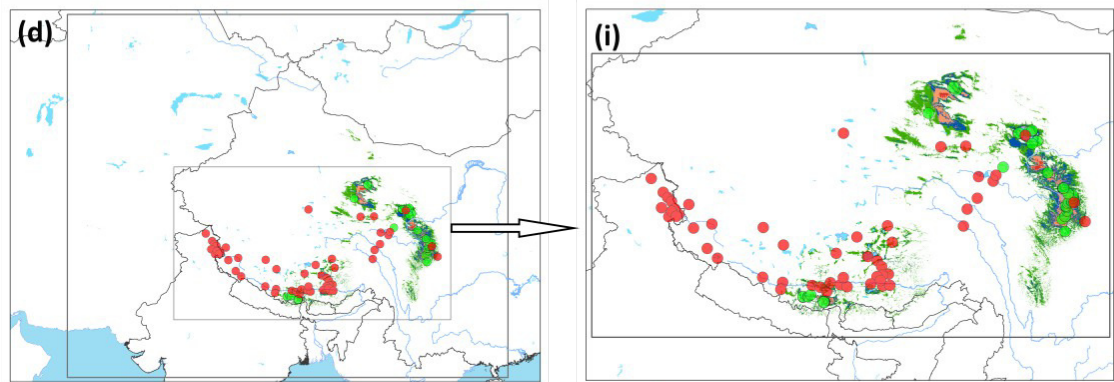

CART
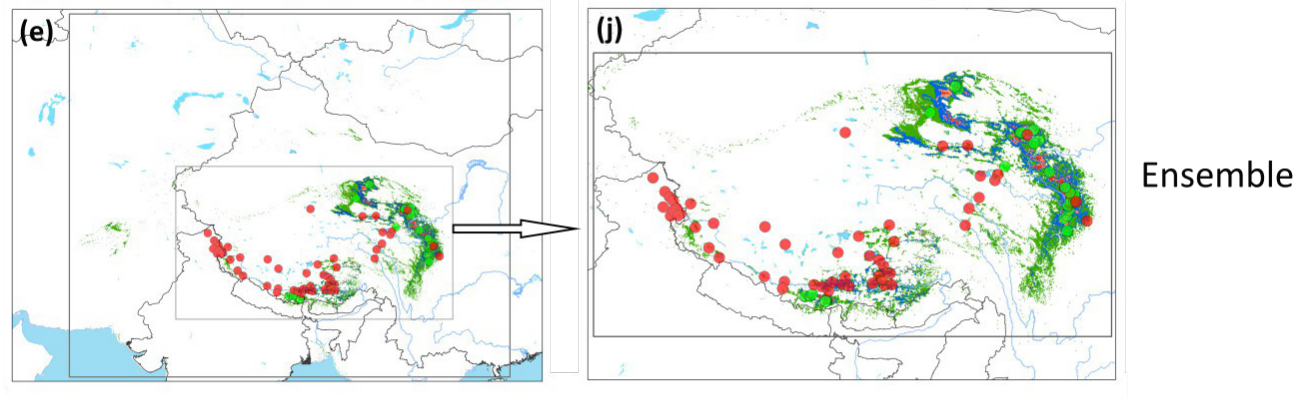

Field nest data $(n=75) \quad$ Literature data $(n=53)$

$0.20-0.40$

$0.40-0.60$

$0.60-0.80$

$0.80-1.00$

Maxent

Ensemble

279 Figure 7 Prediction maps for Black-necked Cranes and zoomed-in maps showing the four models

280 (TreeNet, Random Forest, CART and Maxent) and ensemble model in detail. 7a-7e) prediction map for 
Black-necked Cranes, 7f-7j) zoomed-in map for Black-necked Cranes.

\section{DISCUSSION}

\section{Model generality (transferability)}

284 Estimating species distributions in undersampled areas is a fundamental problem in ecology, 285 biogeography, biodiversity conservation and natural resource management (Drew et al., 2011). 286 That is specifically true for rare and difficult to be detected species and which are usually high on 287 the conservation priority. The use of SDMs and with machine learning has become the method 288 for deriving such estimates (Guisan \& Thuiller, 2005; Drew et al., 2011; Guisan et al., 2013) and 289 could contribute to detect new and to confirm populations of rare species. However, the application of a few samples to project a distribution area widely beyond the sample range is a greater challenge and has rarely been attempted in the literature until recently. And only now have conservationists realized its substantial value for pro-active decision making in conservation management (see work by Ohse et al., 2010; Drew et al., 2011; Kandel et al., 2015 etc.). Our results based on AUC, violin plots for RIOs and spatial assessment of testing data (satellite tracking data and literature data) all suggest there are difference in the generalization performance of different modeling techniques (TreeNet, Random Forest, CART and Maxent).

297 Moreover, among the acknowledged four rather powerful and commonly used machnelearning techniques, Random Forest (bagging) in SPM usually had the best performance in each case. Our results are in agreement with those of Prasad et al. (2006), Cutler et al. (2007) and Syphard and Franklin (2009) indicating a superiority of Random Forest in such applications. However, initially it appears to run counter to the conclusions off recent paper (Heikkinen et al., 2012) with the poor transferability of Random Forest. But we propose this is due to the fact that many Random Forest implementations exist (see the 100 classifier paper Fernández-Delgado et al., 2014). 
305 Here we applied Random Forest in SPM which has been optimized under one of the 306 algorithm's original co-authors, while Heikkinen et al. (2012) run a basic Random Forest with 307 BIOMOD framework in the R sofeware and which remains widely un-tuned and largely behind 308 the potential. The differences are known to be rather big (see Herrick 2013 for a comparison).

309 Furthermore, Maxent, a widely used SDM-method consequently greatly enjoyed by many 310 modelers (Phillips et al., 2006; Peterson et al. 2007; Phillips and Dudík 2008; Li et al., 2015, 311 etc.), didn't perform so good in regards to transferability in this study. This contrasts to those of 312 Elith et al. (2006) and Heikkinen et al. (2012), where Maxent and GBM perform well. We infer 313 this may be caused by sample size used as training data and due to the actual algorithms used. 314 When the sample size increased (33 to 75), the AUC and TSS value of all models rose (Table 2). 315 This indicates that higher sample sizes make models more robust and performing better. Sample 316 sizes of 33 presence points still favor by Random Forest.

317 In Random Forest, random samples from rows and predictors are used to build hundreds of 318 trees. Each individual tree is constructed from a bootstrap sample and split at each node by the 319 best predictor from a very small, randomly chosen subset of the predictor variable pool (Herrick, 320 2013). These trees comprising the forest are each grown to maximal depth, and predictions are made by averaged trees through 'voting' (Breiman et al., 2006). This algorithm avoids overfitting by controlling the number of predictors randomly used at each split, using means of out-of-bag (OOB) samples to calculate an unbiased error rate. And also, Random Forest in SPM utilizes additional specific fine-tuning for best performance.

\section{RIOs of random points}

In order to explore whether Random Forest created higher RIOs for prediction maps in each grid, 
329 related projected study areas. We made violin plots for RIOs of random points (Fig. 8), and we 330 found that more RIO values of random points for Maxent, Random Forest and ensemble models 331 were close to the lower value, and then followed by TreeNet. The distribution shapes of Random 332 Forest, Maxent and ensemble model are more similar to the real distribution of species in the real 333 world. The RIOs of White-naped Crane extracted from the CART model distributed in the range 334 of the low value. That means there were no points located in the high RIO areas of cranes, and 335 which is unrealistic. Consequently, we argued that Random Forest did not create higher RIOs for 336 prediction maps in each grid in our study. 


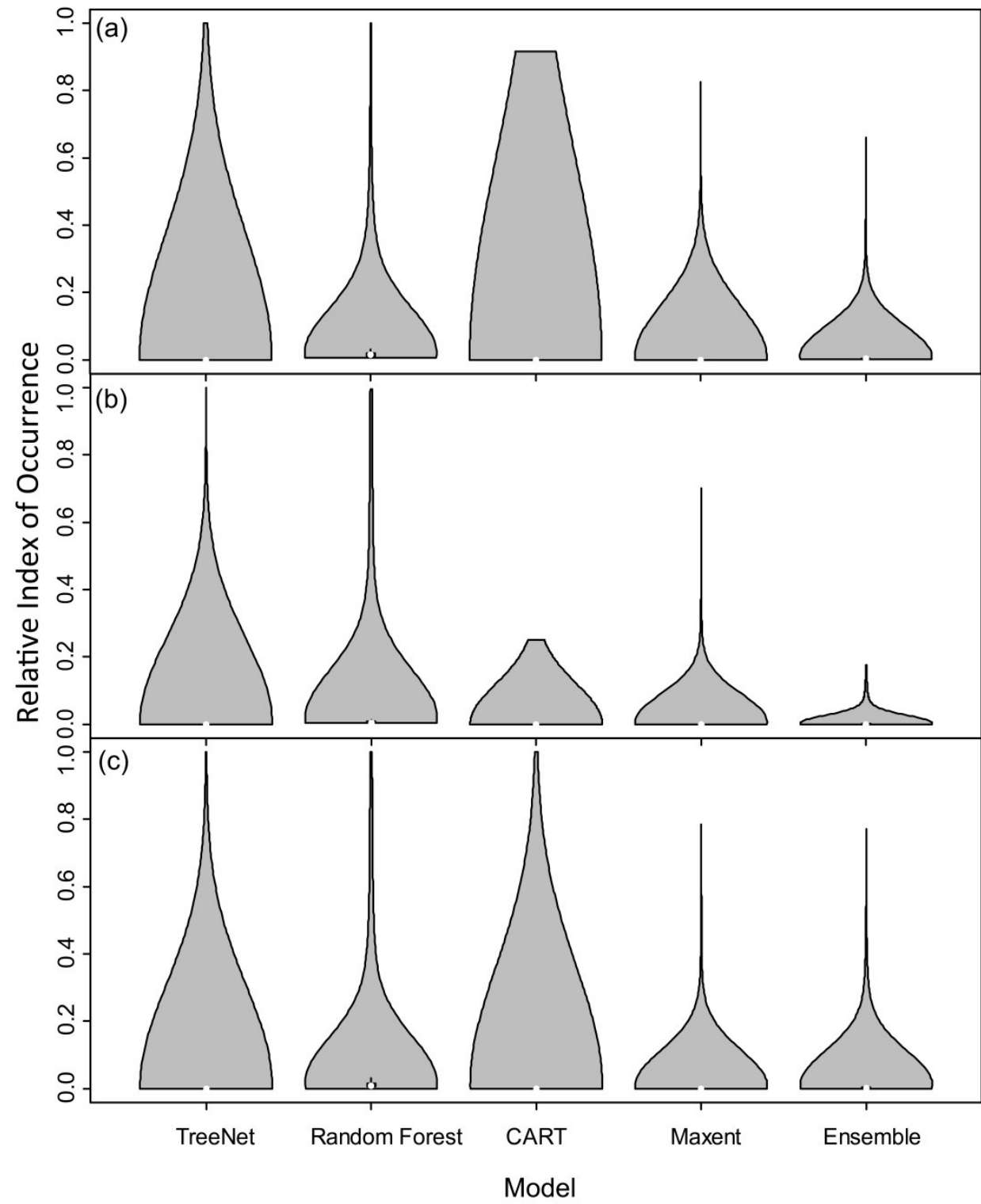

Hooded Crane

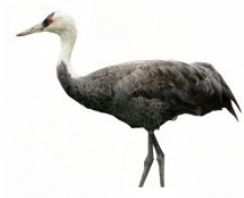

White-naped Crane

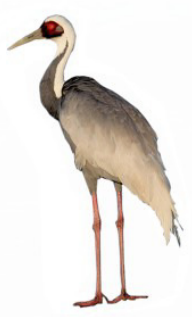

Black-necked Crane

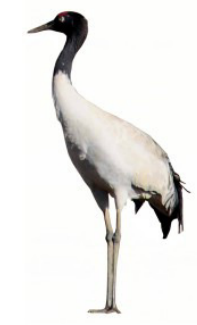

Figure 8 Violin plots of Relative Index of Occurrence (RIO) values for four SDMs and ensemble model for three cranes based on calibration data from Threatened Birds of Asia. 4a) Violin plots for Hooded Cranes, 4b) violin plots for White-naped Cranes, 4c) violin plots for Black-necked Cranes.

343 Conservation biologists are often interested in rare species and seek to improve their conservation. These species typically have limited number of available occurrence records, which 
345 poses challenges for the creation of accurate species distribution models when compared with 346 models developed with greater numbers of occurrences (Stockwell \& Peterson, 2002; McPherson 347 et al., 2004; Hernandez et al., 2006). In this study, we used three crane species as case studies, 348 and their occurrence records (nests) totaled 33, 40, and 75, respectively (considering the small numbers of samples and given that a low fraction of the area was sampled in the large projected area). In our models, we found that model fit (AUC and TSS, see Table 2) of Random Forest that had the highest index, while Maxent usually ranked second. In addition, we found that models with few presence samples can also generate accurate species predictive distributions (Fig. 3 to 7 ) with the Random Forest method. Of course, models constructed with few samples underlie the threat of being biased more because few samples usually had not enough information including all distribution gradients conditions of a species, especially for places far away from the location of training presence points. However, the potential distribution area predicted by SDMs could become the place where scholars could look for the birds (additional fieldwork sampling). And also, these places could be used as diffusion or reintroduction areas! It's valuable and new information either way.

\section{Evaluation methods}

In this study, we applied two widely-used assessment methods (AUC and TSS) in SDMs (Table 2). For an evaluation of these three values we used the approach recommended by Fielding \& Bell (1997), and Allouche et al. (2006), we found our model usually didn't obtain perfect performance, and some of them were 'fair' in their performance. However, for macro-ecology this is more than reasonable and ranks rather high. It's good conservation progress! We identified Random Forest as always the highest performing. These results are consistent with the results of violin plots of the Relative Index of Occurrence (RIO) using tracking as well as literature data

(Figs 3 and 4), and well as matching the spatial assessment results (Figs 5-7). And we 
369

370

371

372

373

374

375

376

377

378

379

380

381

recommend when modelers assess model performance they should not only depend alone on some metric (such as AUC and TSS), but also should base their assessments on the combined use of visualization and expert knowledge. That means modelers should also assess how the species distribution map actually looks and how it links with real data (see Huettmann \& Gottschalk 2011). Spatial assessment metrics from alternative data should matter the most. Expert experience and ecological common knowledge of the species of interest could sometimes also be highly effective (Drew \& Perera, 2011), albeit nonstandard, evaluation methods (see Kandel et al., 2015 for an example). Additionally, one alternative method for rapid assessment we find is to use a reliable SDM, and thus Random Forest would be a good choice in the future given our consistent results (Fig.s 3 to 7, Tables 3 to 5) in this study, which involved three species, a vast landscape to conserve, and only limited data. Our work certainly helps to inform conservation decisions for cranes in Northeast Asia.

\section{Limitations and future work}

Our study is not without limitations: 1) so far, only three species of cranes are used as a test case in our study. That's because nest data for rare species in remote areas are usually sparse; 2) all our species study areas are rather vast and confined to East-Asia. For future work, we would apply

Random Forest in more species and in more geographical conditions with differently distributed features for a first rapid assessment and baseline to be mandatory for better conservation e,g, by governments, IUCN and any impact and court decision. Then we would apply our prediction results in specifically targeted fieldwork sampling campaigns and assess the model accuracy with field survey results (ground-truthing) and with new satellite tracking and drone data, for instance. This is to be fed directly into the conservation management process.

\section{ACKNOWLEDGEMENTS}

We thank Fengqin Yu, Yanchang Gu, Linxiang Hou, Jianguo Fu, Bin Wang, Jianzhi Li, Lama 
393 Tashi Sangpo, Baiyu Lamasery, and Nyainbo Yuze for their hard work in the field. Thanks to all 394 data contributors to the book 'Threatened Birds of Asia'. Thanks to the support of State Forestry 395 Administration and Whitley Fund for Nature (WFN). Further we thank Salford Systems Ltd. 396 (Dan Steinberg) for providing the free trial version of their data mining and machine learning 397 software to the conservation research community. This is EWHALE lab publication \#177. 
398 REFERENCES

399 Allouche OA, Tsoar, Kadmon R. 2006. Assessing the accuracy of species distribution models:

400 prevalence, kappa and the true skill statistic (TSS). Journal of Applied Ecology 43(6):1223-

$401 \quad 1232$.

402

403

404

405

406

407

408

409

410

411

412

413

414

Araújo MB, New M. 2007. Ensemble forecasting of species distributions. Trends in Ecology \& Evolution 22(1):42-47.

Araújo MB, Whittaker R, Ladle R, Erhard M. 2005. Reducing uncertainty in projections of extinction risk from climate change. Global Ecology \& Biogeography 14(6):529-538.

Ashtonw C, Perera AH. 2010. Expert Knowledge as a Basis for Landscape Ecological Predictive Models. Predictive Species \& Habitat Modeling in Landscape Ecology:229-248.

Beyer H. 2013. Hawth's Analysis Tools for ArcGIS version 3.27 (software). in.

Booms TL, Huettmann F, Schempf PF. 2010. Gyrfalcon nest distribution in Alaska based on a predictive GIS model. Polar biology 33(3):347-358.

Boyce MS, Vernier PR, Nielsen SE, Schmiegelow FK. 2002. Evaluating resource selection functions. Ecological Modelling 157(2-3):281-300.

Braunisch V, Coppes J, Arlettaz R, Suchant R, Schmid H, Bollmann K. 2013. Selecting from correlated climate variables: a major source of uncertainty for predicting species distributions under climate change. Ecography 36(9):971-983.

Breiman L. 2001. Random forests. Machine learning 45(1):5-32.

Breiman L., Friedman J, Stone CJ, Olshen RA. 1984. Classification and regression trees. CRC press.

Bucklin DN, Basille M, Benscoter AM, Brandt LA, Mazzotti FJ, Romanach SS, Speroterra C, Watling JI. 2015. Comparing species distribution models constructed with different subsets of environmental predictors. Diversity and Distributions 21(1):23-35. 
422

423

424

425

426

427

428

429

430

Cohen J. 1960. A Coefficient of Agreement for Nominal Scales. Educational and Psychological Measurement 20(1):37-46.

Collar NJ, Crosby R, Crosby M. 2001. Threatened birds of Asia: the BirdLife International red data book. Volume 1.BirdLife International Cambridge, UK.

Cushman SA, Huettmann F. 2010. Spatial Complexity, Informatics, and Wildlife Conservation. Springer, Springer Tokyo Berlin Heidelberg New York.

Cutler DR, Edwards Jr TC, Beard KH, Cutler A, Hess KT, Gibson J, Lawler JJ. 2007. Random forests for classification in ecology. Ecology 88(11):2783-2792.

Drew CA, Perera AH. 2011. Expert knowledge as a basis for landscape ecological predictive models. Pages 229-248 in Predictive Species and Habitat Modeling in Landscape Ecology. Springer.

Drew CA, Wiersma Y, Huettmann F. 2011. Predictive species and habitat modeling in landscape ecology. Springer.

Elith J, Graham CH, Anderson RP, Dudík M, Ferrier S, Guisan A, Hijmans RJ, Huettmann F, Leathwick JR, Lehmann A, Li J, Lohmann LG, Loiselle BA, Manion G, Moritz C, Nakamura M, Nakazawa Y, Overton JMM, Peterson AT, Phillips SJ, Richardson K, Scachetti-Pereira R, Schapire RE, Soberón J, Williams S, Wisz MS, Zimmermann NE. 2006. Novel methods improve prediction of species' distributions from occurrence data. Ecography 29(2):129-151.

Eskildsen, A., P. C. Roux, R. K. Heikkinen, T. T. Høye, W. D. Kissling, J. Pöyry, M. S. Wisz, and M. Luoto. 2013. Testing species distribution models across space and time: high latitude butterflies and recent warming. Global ecology and biogeography 22(12):1293-1303.

Estes L, Bradley B, Beukes H, Hole D, Lau D, Oppenheimer M, Schulze R, Tadross M, Turner W. 2013. Comparing mechanistic and empirical model projections of crop suitability and productivity: implications for ecological forecasting. Global ecology and biogeography 22(8):1007-1018. 
447 Fernández-Delgado M, Cernadas E, Barro S, Amorim D. 2014. Do we need hundreds of 448 classifiers to solve real world classification problems? The Journal of Machine Learning $449 \quad$ Research 15(1):3133-3181.

450 Ferrier S, Watson G, Pearce J, Drielsma M. 2002. Extended statistical approaches to modelling 451 spatial pattern in biodiversity in northeast New South Wales. I. Species-level modelling. 452 Biodiversity \& Conservation 11(12):2275-2307.

453 Fielding AH, Bell JF. 1997. A review of methods for the assessment of prediction errors in 454 conservation presence/absence models. Environmental conservation 24(1):38-49.

455 Fielding AH, Haworth PF. 1995. Testing the Generality of Bird $\square$ Habitat Models. Conservation 456 biology 9(6):1466-1481.

457 Ferrier S, Watson G, Pearce J, Drielsma M. 2002. Extended statistical approaches to modelling 458 spatial pattern in biodiversity in northeast New South Wales. I. Species-level modelling. $459 \quad$ Biodiversity \& Conservation 11(12):2275-2307.

460 Friedman JH. 2002. Stochastic gradient boosting. Computational Statistics \& Data Analysis $461 \quad 38(4): 367-378$.

462 Graham CH, Ferrier S, Huettman F, Moritz C, Peterson AT. 2004. New developments in museumbased informatics and applications in biodiversity analysis. Trends in Ecology \& Evolution 19(9):497-503.

Guillera $\square$ Arroita G, Lahoz $\square$ Monfort JJ, Elith J, Gordon A, Kujala H, Lentini PE, McCarthy 
distributions for conservation decisions. Ecology letters 16(12):1424-1435.

473 Guisan A, Zimmermann NE. 2000. Predictive habitat distribution models in ecology. Ecological $474 \quad$ Modelling 135(2):147-186.

475 Gwena LL, Robin E, Erika F, Guisan A. 2010. Prospective sampling based on model ensembles 476 improves the detection of rare species. Ecography 33(6):1015-1027.

Hanley JA, McNeil BJ. 1982. The meaning and use of the area under a receiver operating 478 characteristic (ROC) curve. Radiology 143(1):29-36.

Hanley JA, McNeil BJ. 1983. A method of comparing the areas under receiver operating characteristic curves derived from the same cases. Radiology 148(3):839-843.

Hijmans RJ, Graham CH. 2006. The ability of climate envelope models to predict the effect of

Hernandez PA, Graham CH, Master LL, Albert DL. 2006. The effect of sample size and species distribution models come at the expense of transferability? Ecography 35(3):276-288.

Hegel TM, SA Cushman, J Evans, Huettmann F. 2010. Current State of the Art for Statistical Modelling of Species Distributions. Spatial Complexity, Informatics, and Wildlife Conservation:273-311.

Heikkinen RK, Marmion M, Luoto M. 2012. Does the interpolation accuracy of species characteristics on performance of different species distribution modeling methods. Ecography 29(5):773-785.

Herrick K. 2013. Predictive Modeling of Avian Influenza in Wild Birds. Veterinary Research. climate change on species distributions. Global Change Biology 12(12):2272-2281.

Huettmann F, Gottschalk T. 2011. Simplicity, Model Fit, Complexity and Uncertainty in Spatial 
497

498

499

500

501

502

503

504

505

506

507

508

509

510

511

512

513

514

515

516

517

518

519

520

521

Prediction Models Applied Over Time: We Are Quite Sure, Aren’t We? Pages 189-208 in

Predictive Species and Habitat Modeling in Landscape Ecology. Springer.

Humphries GRW, Huettmann F. 2014. Putting models to a good use: a rapid assessment of Arctic seabird biodiversity indicates potential conflicts with shipping lanes and human activity. Diversity and Distributions 20(4):478-490.

Jiguet F, Barbet-Massin M, Chevallier D. 2011. Predictive distribution models applied to satellite tracks: modelling the western African winter range of European migrant Black Storks Ciconia nigra. Journal of Ornithology 152(1):111-118.

Kandel K, Huettmann F, Suwal MK, Regmi GR, Nijman V, Nekaris K, Lama ST, Thapa A, Sharma HP, Subedi TR. 2015. Rapid multi-nation distribution assessment of a charismatic conservation species using open access ensemble model GIS predictions: Red panda (Ailurus fulgens) in the Hindu-Kush Himalaya region. Biological Conservation 181:150-161.

Keith DA, Elith J, Simpson CC. 2014. Predicting distribution changes of a mire ecosystem under future climates. Diversity and Distributions 20(4):440-454.

Kessler A, Batbayar N, Natsagdorj T, Batsuur D, Smith A. 2013. Satellite telemetry reveals long $\square$ distance migration in the Asian great bustard Otis tarda dybowskii. Journal of Avian Biology 44(4):311-320.

Kleyer M. 2002. Validation of plant functional types across two contrasting landscapes. Journal of Vegetation Science 13(2):167-178.

Lei, Z., L. Shirong, S. Pengsen, and WangTongli. 2011. Comparative evaluation of multiple models of the effects of climate change on the potential distribution of Pinus massoniana. Chinese Journal of Plant Ecology 35(11):1091-1105.

Li, R., M. Xu, M. H. G. Wong, S. Qiu, X. Li, D. Ehrenfeld, and D. Li. 2015. Climate change threatens giant panda protection in the 21st century. Biological Conservation 182:93-101.

Maggini R, Lehmann A, Zbinden N, Zimmermann NE, Bolliger J, Schröder B, Foppen R, 
Schmid H, Beniston M, Jenni L. 2014. Assessing species vulnerability to climate and land use change: the case of the Swiss breeding birds. Diversity and Distributions 20(6):708-719.

Manel S, Williams HC, Ormerod SJ. 2001. Evaluating presence-absence models in ecology: the need to account for prevalence. Journal of Applied Ecology 38(5):921-931.

McPherson J, Jetz W, Rogers DJ. 2004. The effects of species' range sizes on the accuracy of distribution models: ecological phenomenon or statistical artefact? Journal of Applied Ecology 41(5):811-823.

529 Mi C, Huettmann. F, Guo Y. 2016. Climate envelope predictions indicate an enlarged suitable wintering distribution for Great Bustards (Otis tarda dybowskii) in China for the 21st century.

PeerJ 4:e1630.

Mingchang C, Guangsheng Z, Ensheng W. 2005. Application and comparison of generalized models and classification and regression tree in simulating trees species distribution. ACTA ECOLOGICA SINICA 25(8):2031-2040.

Navarro $\square$ Cerrillo R, Hernández $\square$ Bermejo J, Hernández $\square$ Clemente R. 2011. Evaluating models to assess the distribution of Buxus balearica in southern Spain. Applied Vegetation Science 14(2):256-267.

Ohse B, Huettmann F, Ickert-Bond SM, Juday GP. 2009. Modeling the distribution of white spruce (Picea glauca) for Alaska with high accuracy: an open access role-model for predicting tree species in last remaining wilderness areas. Polar biology 32(12):1717-1729.

Pearson RG, Raxworthy CJ, Nakamura M, Peterson AT. 2007. Predicting species distributions from small numbers of occurrence records: a test case using cryptic geckos in Madagascar. Journal of Biogeography 34(1):102-117.

Peterson AT., Monica P, Muir E. 2007. Transferability and model evaluation in ecological niche modeling: a comparison of GARP and Maxent. Ecography 30(4):550-560.

546 Phillips SJ, Anderson RP, Schapire RE. 2006. Maximum entropy modeling of species geographic 
distributions. Ecological Modelling 190(3):231-259.

548 Phillips SJ, Dudík M. 2008. Modeling of species distributions with Maxent: new extensions and a 549 comprehensive evaluation. Ecography 31(2):161-175.

550 Phillips SJ, Dudík M, Schapire RE. A maximum entropy approach to species distribution $551 \quad$ modeling. ACM, 2004.

552 Prasad AM, Iverson LR, Liaw A. 2006. Newer Classification and Regression Tree Techniques: 553 Bagging and Random Forests for Ecological Prediction. Ecosystems 9(2):181-199.

554 Randin CF, Dirnböck T, Dullinger S, Zimmermann NE, Zappa M, Guisan A. 2006. Are niche555 based species distribution models transferable in space? Journal of Biogeography $556 \quad 33(10): 1689-1703$.

Romo H, García-Barros E, Márquez AL, Moreno JC, Real R. 2014. Effects of climate change on the distribution of ecologically interacting species: butterflies and their main food plants in

Stockwell DR, Peterson AT. 2002. Effects of sample size on accuracy of species distribution Spain. Ecography 37(11):1063-1072. models. Ecological Modelling 148(1):1-13.

Stokes KL, Broderick AC, Canbolat AF, Candan O, Fuller WJ, Glen F, Levy Y, Rees AF, Rilov G, Snape RT, Stott I, Tchernov D, Godley BJ. 2015. Migratory corridors and foraging hotspots: critical habitats identified for Mediterranean green turtles. Diversity and Distributions 21(6): $665-674$.

Swets JA 1988. Measuring the accuracy of diagnostic systems. Science 240(4857):1285-1293.

Syphard DA, Franklin J. 2009. Differences in spatial predictions among species distribution modeling methods vary with species traits and environmental predictors. Ecography 32(6):907-918.

Thomas JA, Bovee KD. 1993. Application and testing of a procedure to evaluate transferability of habitat suitability criteria. Regulated rivers 8:285-285. 
572 Thuiller W. 2003. BIOMOD-optimizing predictions of species distributions and projecting 573 potential future shifts under global change. Global Change Biology 9(10):1353-1362.

574 Zhai T, Li X. 2012. Climate change induced potential range shift of the crested ibis based on 575 ensemble models. ACTA ECOLOGICA SINICA 32(8):2361-2370 (in Chinese).

576 Williams JN, Seo C, Thorne J, Nelson JK, Erwin S, O’Brien JM, Schwartz MW. 2009. Using 577 species distribution models to predict new occurrences for rare plants. Diversity and $578 \quad$ Distributions 15(4):565-576.

579 Wisz MS, Hijmans RJ, Li J, Peterson AT, Graham CH, Guisan A. 2008. Effects of sample size on 580 the performance of species distribution models. Diversity and Distributions 14(5):763-773.

581 Yen P, Huettmann F, Cooke F. 2004. A large-scale model for the at-sea distribution and 582 abundance of Marbled Murrelets (Brachyramphus marmoratus) during the breeding season in 583 coastal British Columbia, Canada. Ecological Modelling 171(4):395-413.

584 Young N, Carter L, Evangelista P. 2011. A MaxEnt Model v3.3.3e Tutorial.

585 Zhang M, Zhou Z, Chen W, Cannon CH, Raes N, Slik JWF. 2014. Major declines of woody plant 586 species ranges under climate change in Yunnan, China. Diversity and Distributions 20(4):405587415. 